\title{
Le problème de l'ordre chez Pascal
}

\section{Dominique Descotes}

\section{(2) OpenEdition}

\section{Journals}

\section{Édition électronique}

URL : https://journals.openedition.org/studifrancesi/38802

DOI : 10.4000/studifrancesi.38802

ISSN : 2427-5856

\section{Éditeur}

Rosenberg \& Sellier

\section{Édition imprimée}

Date de publication : 1 décembre 2004

Pagination : 281-300

ISSN : 0039-2944

\section{Référence électronique}

Dominique Descotes, « Le problème de l'ordre chez Pascal », Studi Francesi [En ligne], 143 (XLVIII | II) | 2004, mis en ligne le 30 novembre 2015, consulté le 19 mai 2021. URL : http://

journals.openedition.org/studifrancesi/38802 ; DOI : https://doi.org/10.4000/studifrancesi.38802

\section{(c) (i) (9)}

Studi Francesi è distribuita con Licenza Creative Commons Attribuzione - Non commerciale - Non opere derivate 4.0 Internazionale. 


\section{Le problème de l'ordre chez Pascal}

Le classement opéré par Pascal dans ses Pensées s'ouvre sur la liasse Ordre: quoiqu'elle ne contienne pas à proprement parler le plan de l'apologie, elle témoigne de la volonté de s'expliquer sur les fondements de la méthode ${ }^{1}$. Est-ce un simple échafaudage appelé à disparaître, ou Pascal comptait-il s'expliquer sur son ordre dans une préface ad hoc? Cette dernière hypothèse n'est pas invraisemblable: l'annonce des différentes étapes du programme de démonstration est un trait constant de sa rhétorique. Achevés ou inachevés, les textes en témoignent. En tête de la quinzième Provinciale, Pascal avertit point par point les Jésuites de ce qu'il entend établir contre eux: «Je ne ferai pas voir seulement que vos écrits sont remplis de calomnies, je veux passer plus avant. On peut bien dire des choses fausses en les croyant véritables, mais la qualité de menteur enferme l'intention de mentir. Je ferai donc voir, mes Pères, que votre intention est de mentir et de calomnier; et que c'est avec connaissance et avec dessein que vous imposez à vos ennemis des crimes dont vous savez qu'ils sont innocents, parce que vous croyez le pouvoir faire sans déchoir de l'état de grâce.» La onzième Provinciale fournirait un exemple tout aussi convaincant. Enfin, dans ses œuvres scientifiques, Pascal procède souvent de même: le premier chapitre de La pesanteur de la masse de l'air est d'une certaine manière le sommaire raisonné du traité entier. Les Pensées, où l'on voit l'ordre à l'état naissant, témoignent aussi du souci du classement concret: «Ordre. Après la lettre qu'on doit chercher Dieu, faire la lettre d'ôter les obstacles qui est le discours de la Machine, de préparer la Machine, de chercher par raison»»».

Ce souci de l'ordre rapproche Pascal de Descartes. L'éloge que L'esprit géométrique accorde à ce dernier porte précisément sur ce point, qu'une idée ne prend son sens véritable que dans l'enchaînement des raisons : malgré les réserves que suscite l'ambition de science universelle, Pascal approuve sans réserve la manière dont Descartes a tenté de tirer par ordre toute les conséquences qu'enferme l'évidence première du cogito: «je sais combien il y a de différence entre écrire un mot à l'aventure, sans y faire une réflexion plus longue et plus étendue, et apercevoir dans ce mot une suite admirable de conséquences, qui prouve la distinction des natures matérielle et spirituelle, et en faire un principe ferme et soutenu d'une physique entière, comme Descartes a prétendu faire». Une pensée qui ne respecte pas l'ordre est mal conçue, et une pensée dont on ne rend pas l'ordre est mal comprise. C'est là-dessus que Pascal reprend le P. Noël: il manque à l'ordre en ce qu'il pose des propositions avant d'avoir défini les termes, et que, sans avoir préalablement défini l'espace vide, la lumière et le mouvement, il prétend «montrer par la nature de ces choses une contradiction manifeste dans ces propositions: que la lumière pénètre un espace vide, et qu'un corps s'y meut avec temps».

Si l'ordre fonde la validité des pensées, il fait aussi leur originalité: la nouveauté du cogito, c'est précisément qu'il fait partie d'une chaîne de raisons. «Qu'on ne dise pas que je n'ai rien dit de nouveau, la disposition des matières est nouvelle. Quand on joue à la paume, c'est une même balle dont joue l'un et l'autre, mais l'un la place mieux. J'aimerais autant qu'on me dise que je me suis servi des mots anciens. Et comme si les mêmes pensées ne formaient pas un autre corps de discours par une disposition différente, aussi bien que les mêmes mots forment d'autres pensées par leur différente disposition». 
L'ordre strict et véritable est le privilège de la géométrie, «la seule des sciences humaines qui produise des démonstrations infaillibles», parce qu'elle suit la véritable méthode. Elle ne possède pas l'ordre parfait, qui consisterait à tout définir et tout prouver, car il est chimérique; mais elle se contente d'une perfection relative et suffisante, qui consiste à définir et à prouver seulement le strict nécessaire, mais tout le nécessaire. Dans toutes les sciences autres que la géométrie, le véritable ordre est difficile, voire impossible à trouver: elles demeurent par «une nécessité naturelle dans quelque sorte de confusion que les seuls géomètres savent extrêmement reconnaître». De son aveu même, Pascal a rencontré ce problème dans la rédaction de son Apologie: «Ordre. J'aurais bien pris ce discours d'ordre comme celui-ci: pour montrer la vanité de toutes sortes de conditions, montrer la vanité des vies communes, et puis la vanité des vies philosophiques, pyrrhoniennes, stö̈ques; mais l'ordre n'y serait pas gardé. Je sais un peu ce que c'est, et combien peu de gens l'entendent. Nulle science humaine ne le peut garder. Saint Thomas ne l'a pas gardé». Il n'attribue pas cette difficulté à sa seule faiblesse: c'est l'effet d'une nécessité naturelle: «Ordre. La nature a mis toutes ses vérités en soi-même. Notre art les renferme les unes dans les autres, mais cela n'est pas naturel. Chacune tient sa place». Les meilleures solutions révèlent vite leur insuffisance: veut-on diordonner la morale «en abstine et sustine plutôt qu'en suivre nature ou faire ses affaires particulières sans injustice comme Platon, ou autre chose». Dès que l'on «vient à l'expliquer, dès qu'on ouvre ce précepte qui contient tous les autres ils en sortent en la première confusion que vous vouliez éviter». Il arrive d'ailleurs que le seul ordre adéquat soit le désordre: pour parler du pyrrhonisme, écrire ses pensées «sans ordre» est «le véritable ordre et qui marquera toujours (son) objet par le désordre même».

Qu'est-ce que l'ordre? Au sens le plus élémentaire, c'est une règle de succession qui détermine la priorité de certaines opérations intellectuelles sur d'autres. La relation qui définit un ordre doit avoir trois propriétés. Elle doit être connective, en ce sens que, si on donne deux termes quelconques, l'un doit précéder et l'autre suivre. Elle doit être asymétrique, c'est-à-dire que si $\mathrm{x}$ précède $\mathrm{y}, \mathrm{y}$ ne peut pas précéder $\mathrm{x}$ : si par exemple $\mathrm{x}<\mathrm{y}$, il est impossible que $\mathrm{x}>\mathrm{y}$. Enfin, elle doit être transitive: si $\mathrm{x}$ précède $\mathrm{y}$, et si y précède $z$, $\mathrm{x}$ précède $\mathrm{z}$ : si $\mathrm{x}>\mathrm{y}$, et si $\mathrm{y}>\mathrm{z}$, alors $\mathrm{x}>\mathrm{z}^{2}$. Dans les Règles pour la direction de l'esprit, Descartes résume cela par la distinction de l'absolu et du relatif: toutes les choses peuvent se classer en séries, en tant qu'elles peuvent être connues l'une par l'autre; les idées proposées les premières doivent être obtenues sans l'aide des suivantes; les notions absolues sont celles qui, se trouvant les premières, ne dépendent pas des suivantes, qui leur sont relatives dans la mesure où elles ne peuvent venir qu'après elles et par elles. Pour Descartes, «le vrai chemin pour bien trouver et expliquer la vérité» va a facilioribus ad difficiliora: le plus aisé à connầtre, ou en termes techniques le notior, n'est pas ce qui exige le moins d'effort, car les premiers principes sont très difficiles à découvrir: c'est ce dont la connaissance est requise pour connaître le reste, parce qu'il est plus simple et les idées qui s'ensuivent plus complexes. Descartes précise l'idée par le modèle des moyennes proportionnelles: dans une proportion géométrique $\frac{a}{b}=\frac{b}{c}$ a est une nature simple, $b$ est le moyen terme par lequel on peut parvenir à connaître c. Dans l'ordre direct, pour connaîre $c$, il faut au préalable connaître $a$ et $b$. Plus difficile est le problème inverse, qui consiste, connaissant a et $\mathrm{c}$, a demander $\mathrm{b}$.

L'Esprit géométrique ne propose pas une réflexion abstraite sur la nature de l'ordre; mais il indique ce qu'il est sous l'angle pratique: la géométrie commence par les indémontrables: les définitions nominales d'abord, puis les axiomes ou principes, que l'on pose préalablement à tout autre discours. Suivent les propositions proprement 
dites, qui enferment une thèse à démontrer. L'art de persuader est un art d'ordonner «pour faire voir la liaison des vérités avec leurs principes soit de vrai, soit de plaisir, pourvu que les principes qu'on a une fois avoués demeurent fermes et sans être jamais démentis». Cet art de persuader, «qui n'est proprement que la conduite des preuves méthodiques parfaites, consiste en trois parties essentielles: à definir les termes dont on doit se servir par des définitions claires: à proposer des principes ou axiomes évidents pour prouver la chose dont il s'agit; et à substituer toujours mentalement dans la démonstration les définitions à la place des définis. La raison de cette méthode est evidente, puis qu'il serait inutile de proposer ce qu'on veut prouver et d'en entreprendre la démonstration, si on n'avait défini clairement tous les termes qui ne sont pas intelligibles; et qu'il faut de même que la démonstration soit précédée de la demande des principes évidents qui y sont nécessaires, car si l'on n'assure le fondement on ne peut assurer l'édifice.»

Ce qui fait l'originalité de la réflexion de Pascal, c'est que le critère de classement n'est pas, comme chez Descartes, la succession logique des idées abstraites. Il se situe dans le domaine de la communication et de l'accord des esprits: dans les Ecrits sur la grâce par exemple, il annonce une progression des choses «qui sont sans contestation», c'est-à-dire sur lesquelles tous les chrétiens sont d'accord, à «celles qui sont contestées» entre catholiques, protestants et pélagiens: les premières conduisent «insensiblement à concevoir» les secondes. Pour ordonner les différentes étapes de son argumentation, Pascal prend aussi en compte les effets psychologiques qu'il veut obtenir: «Ordre. Les hommes ont mépris pour la religion. Ils en ont haine et peur qu'elle soit vraie. Pour guérir cela il faut commencer par montrer que la religion n'est point contraire à la raison. Vénérable, en donner respect. La rendre ensuite aimable, faire souhaiter aux bons qu'elle fût vraie et puis montrer qu'elle est vraie. Vénérable parce qu'elle a bien connu l'homme. Aimable parce qu'elle promet le vrai bien.»

De fait, il existe un certain écart entre L'esprit géométrique, qui ne formule que des règles générales, mais élémentaires, et la pratique concrète de Pascal, qui est plus savante.

Dans ses écrits scientifiques, la progression est en général conforme au modèle euclidien: elle consiste à regrouper en tête de l'ouvrage les définitions et les axiomes pour venir ensuite aux propositions. Dans le Triangle aritbmétique, la construction du Triangle précède tout le reste; et dans les traités suivants, les définitions des combinaisons ou des ordres numériques précèdent les conséquences qui en découlent. Il arrive pourtant que, suivant l'exemple de Ramus et de Stevin, Pascal accommode cet ordre en apportant les définitions à mesure qu'il en a besoin, voire à réserver certaines d'entre elles jusqu'au moment où la démonstration les rendra opportunes et plus faciles à comprendre. Dans la Lettre à Carcavy, qui ouvre ses traités sur la roulette, il donne des le début la définition du concept nouveau de somme triangulaire: «s'il y a tant de quantités qu'on voudra $\mathrm{A}, \mathrm{B}, \mathrm{C}, \mathrm{D}$, lesquelles on prenne en cette sorte : premièrement la somme de toutes, A, B, C, D; puis la somme des mêmes excepté la première, savoir B, C, D; puis la somme des mêmes exceptées les deux premières, C, $\mathrm{D}$, et ainsi toujours, ..., j'appelle la somme de ces quantités, prises en cette sorte, la somme triangulaire de ces mêmes quantités à commencer par A... »

$$
\mathrm{T}_{\mathrm{A}}^{\mathrm{D}}=(\mathrm{A}+\mathrm{B}+\mathrm{C}+\mathrm{D})+(\mathrm{B}+\mathrm{C}+\mathrm{D})+(\mathrm{C}+\mathrm{D})+\mathrm{D}
$$




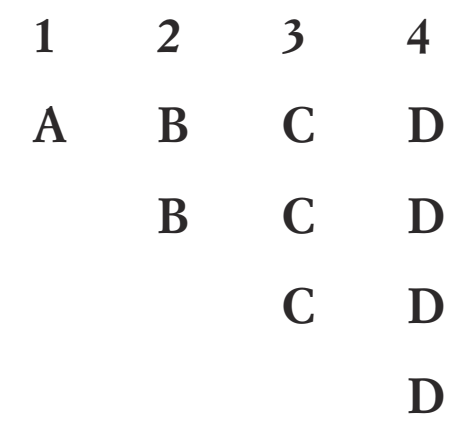

Un lemme montre ensuite que cette manière de prendre la somme triangulaire est équivalente à celle qui consiste à affecter ces grandeurs d'un coefficient croissant répondant à leur rang dans la somme :

$T^{\mathrm{D}}=(\mathrm{A}+\mathrm{B}+\mathrm{C}+\mathrm{D})+(\mathrm{B}+\mathrm{C}+\mathrm{D})+(\mathrm{C}+\mathrm{D})+\mathrm{D}=\mathrm{A}+2 \mathrm{~B}+3 \mathrm{C}+4 \mathrm{D}$

Pascal pourrait, tout de suite après cette définition liminaire, proposer celle de la somme pyramidale, qui est directement connexe, puisque c'est la somme des sommes triangulaires dégressives.

\begin{tabular}{|ccc|}
\hline SOMME & SOMME & SOMME \\
SIMPLE & TRIANGULAIRE & PYRAMIDALE \\
\hline ABC & ABC & ABC \\
& BC & BC \\
& C & C \\
& & BC \\
& & $\mathrm{C}$ \\
& & $\mathrm{C}$ \\
\hline
\end{tabular}

Il préfère retarder cette deuxième définition: comme le concept de somme triangulaire est nouveau, il montre d'abord tout ce que l'on peut en tirer en expliquant en détail, dans de longs Avertissements, comment on forme des sommes triangulaires de lignes, de plans ou de solides; ce n'est qu'après avoir familiarisé le lecteur avec cette pratique qu'il propose la définition des sommes pyramidales, qui n'est pas moins nouvelle, et s'avère d'un maniement plus difficile. En bousculant un peu l'ordre conventionnel de la géométrie, Pascal permet à son lecteur de s'exercer sur la premier type de somme à l'utilisation du second.

Une construction préalable précède souvent les définitions. Dans la Generatio conisectionum, les termes de parabole, d'byperbole et d'ellipse (Pascal dit antobole), bien connus à l'époque, pouvaient à la rigueur se passer de définition. A défaut, ils auraient pu être définis dès les premières lignes. Pascal procède pourtant autrement: il commence par le commencement, avec la construction du cône, dont il dégage les propriétés fondamentales, et dans un second temps seulement, il détermine les différentes manières dont la surface conique peut être coupée par un plan, ce qui amène à la définition des différentes sections. Mais ce n'est pas par plaisir, ni par une vaine volonté d'exhaustivité, qu'il ménage ainsi la présentation des coniques, car contraire- 
ment à ce que l'on pourrait croire, l'essentiel n'est pas dans les courbes, mais dans la construction: bientôt un nouveau corollaire l'intègre dans un modèle perspectiviste, par les concepts de tableau, d'œil et d'image; et c'est par là seulement qu'apparaît la véritable nature des sections coniques, comme images du cercle différant selon la position relative du cône et du plan, ainsi que leurs propriétés respectives, que résume le tableau comparatif final. Cette présentation des coniques vise à faire admettre au lecteur certaines notions qui, présentées de but en blanc, froisseraient son bon sens: il faut en effet que des concepts comme le point à l'infini aient été préalablement introduits, pour que le lecteur comprenne que les deux branches de l'hyperbole ne font en réalité qu'une seule courbe, ou a fortiori qu'il digère des contradictions apparentes comme l'assimilation entre asymptote et tangente à l'infini.

L'opération de division tient chez Descartes une place importante pour la distinction des éléments à ordonner. Il semble à première vue que Pascal lui soit hostile. Leur défaut est qu'elles n'en finissent pas:

«La diversité est si ample que tous les tons de voix, tous les marchers, toussers, mouchers, éternuements. On distingue des fruits les raisins, et entre ceux-là les muscats, et puis Condrieu, et puis Desargues, et puis cette ente. Est-ce tout? en a-t-elle jamais produit deux grappes pareilles, et une grappe a-t-elle deux grains pareils, etc...» «Diversité. La théologie est une science, mais en même temps combien est-ce de sciences? Un homme est un suppôt, mais si on l'anatomise que sera-ce? la tête, le cœur, l'estomac, les veines, chaque veine, chaque portion de veine, le sang, chaque humeur de sang. Une ville, une campagne, de loin c'est une ville et une campagne, mais à mesure qu'on s'approche, ce sont des maisons, des arbres, des tuiles, des feuilles, des herbes, des fourmis, des jambes de fourmis, à l'infini. Tout cela s'enveloppe sous le nom campagne.» Il semble qu'elles soient vouées à l'inefficacité: « Pourquoi prendrai-je plutôt à diviser ma morale en 4 qu'en 6. Pourquoi établirai-je plutôt la vertu en 4 , en 2, en 1 . Pourquoi en abstine et sustine plutôt qu'en suivre nature ou faire ses affaires particulières sans injustice comme Platon, ou autre chose. Mais voilà, direz-vous, tout renfermé en un mot: oui mais cela est inutile si on ne l'explique. Et quand on vient à l'expliquer, dès qu'on ouvre ce précepte qui contient tous les autres ils en sortent en la première confusion que vous vouliez éviter. Ainsi quand ils sont tous renfermés en un ils y sont cachés et inutiles, comme en un coffre et ne paraissent jamais qu'en leur confusion naturelle. La nature les a tous établis, sans renfermer l'un en l'autre.» Enfin, les divisions à la manière de Charron «attristent et ennuient». Pourtant Pascal y recourt souvent. Le projet de plan apologétique en deux parties repose sur une division toute simple: «Préface de la première partie. Parler de ceux qui ont traité de la connaissance de soi-même (...). Préface de la seconde partie. Parler de ceux qui (...) entreprennent de parler de Dieu.» La division entre ceux qui parlent de la nature humaine et de ceux qui parlent de Dieu, des moralistes et des apologistes, est bien destinée, en l'occurrence, à commander la structure de l'ouvrage. De la même manière, les derniers chapitres de L'Equilibre des liqueurs sont construits sur une suite des dichotomies tout à fait classique: le chapitre IV porte sur l'équilibre d'une liqueur avec un corps solide partiellement enfoncé dans l'eau, le cinquième sur les corps (non compressibles) qui sont tout enfoncés dans l'eau, le sixième sur les corps compressibles \{non vivants) qui sont dans l'eau, et le septième sur les (corps compressibles) animaux qui sont dans l'eau. Ce qui fait que ces divisions échappent en partie aux reproches que Pascal adresse aux divisions ordinaires, c'est qu'elles prennent toujours place dans un ensemble fini qui engendre les différents termes. Dans la Generatio conisectionum, la genèse des figures s'effectue aussi par dichotomies successives, toujours étroitement limitées: le sommet d'un cône peut être dans un plan ou hors de ce plan, tertium non datur; la surface conique peut avoir deux verticales dans le plan, une seule ou aucune; enfin, si elle n'a aucune verticale dans le 
plan, elle peut avoir deux verticales parallèles au plan, une seule ou aucune. A chacun de ces cas, qui semblent engendrés par un mécanisme combinatoire, correspond une section conique singulière. Le processus de division demeure réglé et limité. Il est alors apte à commander la construction d'un plan ou d'un ordre.

Pour les propositions, l'ordre est aussi en général conforme aux règles énoncées dans L'esprit géométrique. Il faut «que la démonstration soit précédée de la demande des principes qui y sont nécessaires», car «il faut en tout dialogue et discours qu'on puisse dire à ceux qui s'en offensent: de quoi vous plaignez-vous?» Les propositions élémentaires ouvrent donc un traité ou un mouvement de ce traité. Ce sont parfois des axiomes, surtout lorsque la matière est nouvelle. Dans la théorie des partis, Pascal commence par poser deux principes généraux. Le premier est que «si un des joueurs se trouve en telle condition que, quoi qu'il arrive, une certaine somme lui doit appartenir en cas de perte et de gain, sans que le hasard la lui puisse ôter, il n'en doit faire aucun parti, mais la prendre entière comme assurée... »; et le second, que «si deux joueurs se trouvent en telle condition que, si l'un gagne, il lui appartiendra une certaine somme, et s'il perd, elle appartiendra à l'autre, si le jeu est de pur hasard et qu'il y ait autant de hasard pour l'un que pour l'autre, et par conséquent non plus de raison de gagner pour l'un que pour l'autre, s'ils veulent se séparer sans jouer, et prendre ce qui leur appartient légitimement, le parti est qu'ils séparent la somme qui est au hasard par la moitié, et que chacun prenne la sienne». Ces principes abstraits ne déterminent ni le nombre des parties gagnées par l'un ou l'autre des joueurs, ni le rapport des parties gagnées par chacun, ni les sommes engagées. En découlent deux Corollaires, qui en tirent la conséquence fondamentale que «si deux joueurs jouent à un jeu de pur hasard, à condition que, si le premier gagne, il lui reviendra une certaine somme, et s'il perd, il lui en reviendra une moindre», le parti peut «se faire en assemblant «les deux sommes de gain et de perte, et que le premier prenne la moitié de cette somme». C'est seulement lorsque, par la suite, Pascal envisage les cas successifs que l'on comprend que le premier principe répond au cas où le joueur A a gagné toutes ses parties, et le joueur B a perdu; de même, on comprend plus tard que le second principe correspond au cas où les deux joueurs sont à égalité, chacun n'ayant qu'une partie à gagner pour emporter le jeu.

Lorsque, comme c'est le cas le plus souvent, la matière n'est pas toute nouvelle, Pascal use de lemmes. Un lemme est «une proposition qui n'est au lieu où elle est, que pour servir de preuve à d'autres qui suivent», et qui demande une confirmation que l'auteur réserve pour plus tard'. Dans le traité des Combinaisons par exemple, où les fondements ne sont pas neufs, après la définition préliminaire, Pascal pose trois lemmes fondamentaux qui serviront de fondement à la suite: un nombre ne se combine point dans un plus petit, un nombre quelconque se combine une fois seulement dans son égal, et l'unité se combine dans quelque nombre que ce soit autant de fois qu'il contient d'unités.

$$
\begin{array}{lll}
\mathrm{C}_{\mathrm{p}-\mathrm{x}}^{\mathrm{p}}=0 & \text { ou } & \mathrm{C}_{\mathrm{p}}^{\mathrm{p}+1}=0 \\
\mathrm{C}_{\mathrm{p}}^{\mathrm{p}}=1 & \\
\mathrm{C}_{\mathrm{n}}^{\mathrm{l}}=\mathrm{n} &
\end{array}
$$

Ceux-ci sont presque immédiatement évidents; mais le Lemme 4 a besoin d'une substantielle démonstration.

$$
\mathrm{C}_{\mathrm{n}}^{\mathrm{p}}+\mathrm{C}_{\mathrm{n}}^{\mathrm{p}+1}+\mathrm{C}_{\mathrm{n}+1}^{\mathrm{p}+1}=0
$$

(3) A.ARnauld, Nouveaux Eléments de Géométrie, Euvres Lausanne, 1775-1783, XLII, p. 15. 
Pascal souligne d'ailleurs parfois qu'il est conscient de paraître enfoncer des portes ouvertes, par exemple lorsqu'il envisage le cas où «à un joueur il ne manque aucune partie, et à l'autre quelques-unes», auquel cas «la somme entière appartient au premier». Il explique: «je le fais pourtant pour commencer par le commencement». Mais il ne faut pas trop se fier au caractère apparemment évident ou facile de ces lemmes. Bien souvent, leur simplicité fait oublier qu'ils sont la pierre angulaire de tout l'édifice. Jean Mesnard a montré, sur le Traité des sinus du quart de cercle, que le lemme initial, dans lequel Leibniz a découvert son triangle caractéristique, a été ajouté après la première rédaction, pour en souligner l'importance. La portée de ces premières propositions est parfois déterminante, et les effets de surprise qui en découlent parfois peuvent être saisissants.

On peut proposer pour exemple le Lemme général du Traité des Trilignes, où Pascal s'inspire de Grégoire de Saint-Vincent: sur le triligne BAH et sur le triligne adjoint $\mathrm{BAC}$, les ordonnées $\mathrm{EG}$ et $\mathrm{DF}$ divisent respectivement $\mathrm{AC}$ et $\mathrm{AB}$ en portions égales EE et DD. Des points $\mathrm{G}$ déterminés sur la courbe par les ordonnées EG naissent des contre-ordonnées $\mathrm{GR}$, abaissées sur l'axe $\mathrm{AB}$. Lorsque l'on dresse la figure adjointe $\mathrm{BAC}$ à angle droit par rapport à $\mathrm{BAH}$, les perpendiculaires aux deux trilignes engendrent des surfaces qui délimitent le solide BACH. Ce solide est alors coupé par deux ordres de plans verticaux perpendiculaires au triligne BAC. Le premier est composé par des rectangles (FD.DO) construits sur les ordonnées DF à l'axe $A B$, et sur les droites DO parallèles à AH: multipliés chacun par une portion DD de AB, ils constituent des parallélépipèdes (FD.DO.DD). Le second ordre de plans est parallèle au plan du triligne adjoint BAH. Aux points $\mathrm{R}$ des contre-ordonnées $\mathrm{RG}$, sont élevées verticalement les lignes RI, parallèles entre elles, qui délimitent sur la figure adjointe des portions ARIH. Ces portions ARIH vont se trouver chacune transposées sur la droite EG perpendiculaire à AC correspondante: elles ont ainsi chacune leur représentante placée à une division E de AC. Le solide entier, que Grégoire de Saint Vincent appelle ductus plani in planum, peut ainsi être présenté aussi bien comme la somme des parallélépipèdes (FD.DO.DD) que comme celle des espaces ARIH, chacun doté d'une épaisseur EE, $\Sigma$ (ARIH.EE). Le Lemme établit que ces deux sommes sont égales entre elles, parce qu'elles composent le même solide BACH.
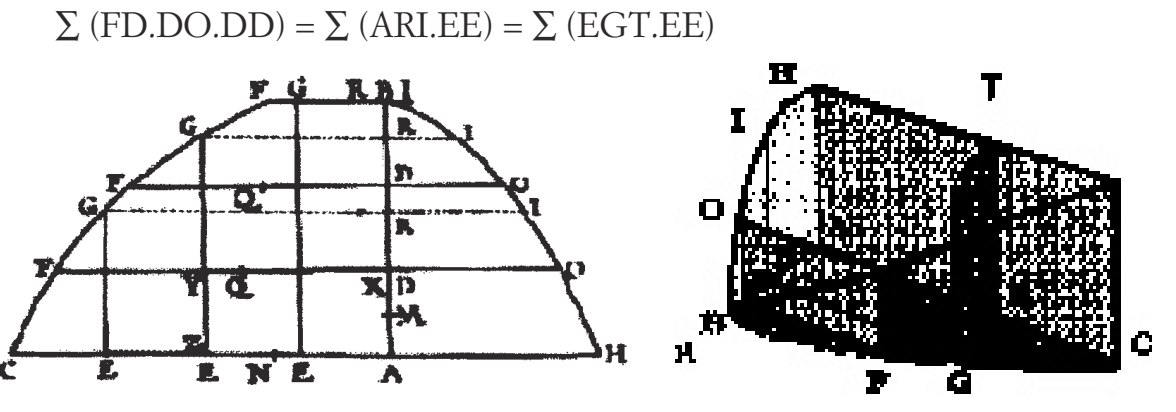

Les propositions qui suivent ce lemme général naissent de l'introduction d'un simple élément de variation, en transformant la figure adjointe $\mathrm{BAH}$, de manière à métamorphoser le solide engendré: lorsque l'adjointe est un triangle isocèle $A B K$, égal à la moitié du carré de même côté, on obtient un onglet dans lequel les hauteurs DO seront toujours égales aux distances AD. On peut le considérer comme la somme des plans rectangulaires (FD.DO.DD) ou (FD.DA.DD). Le lemme précédent s'applique directement, établissant l'égalité de la somme des rectangles FD. DO et celle des (EGT.EE), c'est-à-dire de la moitié des carrés des EG, soit (EG ${ }^{2}$.EE). D'autres métamorphoses 
des figures adjointes engendrent de manière semblable de nouvelles égalités, entre les puissances successives des DF et des EG. Or ces nouvelles propositions dépassent rapidement les trois dimensions de la géométrie naturelle, et engendrent des solides à quatre, cinq ou six dimensions, inconcevables concrètement, ce qui n'a pas manqué de susciter les protestations scandalisées des puristes comme le P. Lalouvère.
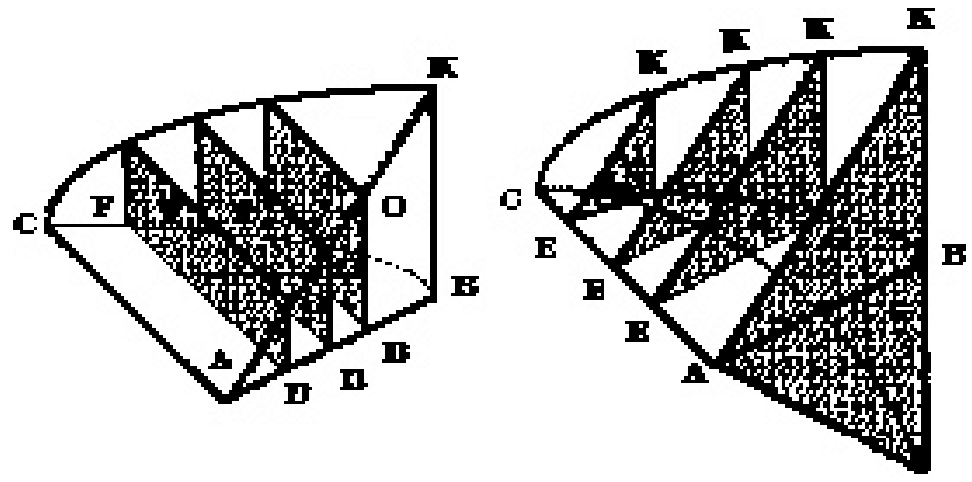

L'argument du pari est construit sur ce modèle. Les propositions initiales forment un cadre axiomatique qui semble aller de soi, dans la mesure où elles obéissent à la règle d'homogénéité du connaissant et du connu: par raison naturelle, nous connaissons l'existence et la nature du fini, parce que nous sommes finis et étendus comme lui; nous connaissons l'existence de l'infini mais non sa nature, parce qu'il a étendue comme nous, mais pas de bornes; enfin, nous ne connaissons ni l'existence ni la nature de Dieu, parce qu'il n'a ni étendue, ni bornes. Ces propositions préliminaires ont des conséquences différentes selon le point de l'ordre des raisons auquel on se place. A un premier stade, elles apparaissent comme une concession faite à l'athée, auquel Pascal accorde que la raison est incapable de démontrer l'existence de Dieu. En deuxième instance pourtant, une fois que l'apologiste a déclaré que le chrétien, par la foi, connaît «qu'il y a un Dieu sans savoir ce qu'il est», le second principe sert à confirmer qu'il n'y a rien d'absurde dans cette prétention, puisque c'est ce qui arrive dans le cas de l'infini mathématique; autrement dit, il disculpe le chrétien de commettre un paralogisme. Ces différentes conséquences, quoique contenues en germe dans les principes, ne sont pas visibles également au premier abord.

D'autre part, il en va des principes comme des définitions: Pascal ne les énonce pas tous d'entrée. L'ordre en fait apparaître progressivement plusieurs, qui viennent s'ajouter aux précédents et les féconder. Dans le pari, c'est le cas au moment où Pascal, pour récuser l'objection que le juste est de ne point parier, répond en énonçant un axiome qu'il n'avait pas encore invoqué: il faut parier, ce n'est pas volontaire. Ce nouveau principe vient indirectement du fragment Laf. 158, Sel . 190: «Par les partis vous devez vous mettre en peine de rechercher la vérité, car si vous mourez sans adorer le vrai principe vous êtes perdu.» Il n'a de force que parce que l'on a intégré l'incertitude sur le fait de savoir si Dieu est ou s'il n'est pas: sachant qu'en cas d'erreur, on risque un malheur éternel, il devient impératif de choisir, et l'abstention n'est pas possible. Mais ce principe admis, l'orientation de l'argumentation change: on ne discutera plus la nécessité du pari, mais du choix qu'il faut faire.

L'enchaînement des conséquences suit en général l'ordre géométrique. Le premier chapitre de La pesanteur de la masse de l'air présente une progression particulièrement stricte. Ayant posé le principe «que l'air est pesant», Pascal poursuit en tirant par ordre «quelques conséquences». Primo, en vertu de l'homogénéité de la partie et du tout, «puisque chaque partie de l'air est pesante, il s'ensuit que la masse entière de l'air ... 
est pesante». Secundo, de même que «la masse de l'eau de la mer presse par son poids la partie de la terre qui lui sert de fond... », «ainsi la masse de l'air couvrant toute la surface de la terre, ce poids la presse en toutes les parties». Tertio, «comme le fond d'un seau où il y a de l'eau» est d'autant plus pressé par le poids de l'eau «qu'il y a plus de hauteur d'eau », «aussi les lieux élevés, comme les sommets des montagnes, ne sont pas si pressés par le poids de la masse de l'air que les lieux profonds, comme les vallons...». Quarto, «comme les corps qui sont dans l'eau sont pressés de toutes parts par le poids de l'eau qui est au-dessus (...), ainsi les corps qui sont dans l'air sont pressés de tous côtés par le poids de la masse de l'air qui est au-dessus». Et ainsi de suite.

Dans l'œuvre mathématique, la Lettre à $M$. ADDS est le meilleur exemple d'un respect strict de la rigueur géométrique. Son objet est de montrer l'égalité des arcs de la parabole et de la spirale d'Archimède. Elle s'ouvre sur des lemmes relatifs aux Propriétés des courbes. Suit l'inscription à chacune d'elles de lignes mixtes inscrites et circonscrites, qui les enserrent chacune entre deux lignes, l'une inférieure, l'autre plus grande, que l'on fera converger en multipliant les points de division du rayon AB. Ensuite, Pascal établit des Rapports entre la parabole et la spirale correspondantes. Enfin, un Problème puis un Théorème montrent que ces lignes ne diffèrent que d'une grandeur moindre que toute donnée; et par suite que les deux courbes elles-mêmes sont égales ${ }^{4}$. Cette exactitude fait contraste avec la nervosité des traités qui procèdent par la méthode des indivisibles. Mais il s'agit là d'un exercice de style, que Pascal ne recommencera pas plus que Racine ne réitérera la tentative de Bérénice.

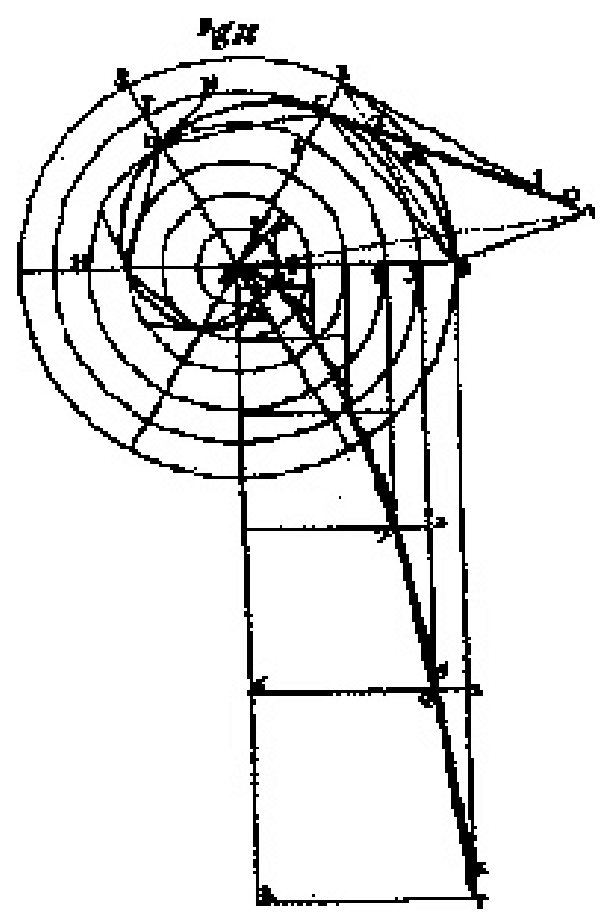

(4) La dernière étape de la Lettre a $M$. ADDS comporte une faute, sans doute due à la contamination de deux rédactions successives. Voir D?:
Descotes, L'argumentation chez Pascal, Paris, Puf, 1993, $153 \mathrm{sq}$. 
Car le respect strict de l'ordre logique des conséquences peut souffrir des modifications et des accommodements en vue de faciliter la compréhension. C'est le cas dans la succession des traités qui composent le Triangle arithmétique. Comme on sait, cet ouvrage a connu deux impressions successives, de construction différente. La première presente une simple juxtaposition de traités consacrés aux applications: le Numeri figurati, le Combinationes, puis le Potestatum numericarum summa. Dans la seconde en revanche, Pascal recourt à un ordre plus éclairant: il détache de chaque traité quelques propositions propres à montrer le rapport de chaque domaine envisagé avec le triangle arithmétique, et il les regroupe dans une section intermédiaire consacrée aux Usages du triangle arithmétique, qui sert d'introduction aux différents traités. Il ajoute même un bref Usage sur les puissances du binôme pour introduire le Potestatum numericarum summa, et un Usage pour les partis. Ces transitions donnent au lecteur une vue d'ensemble sur les différentes manières dont s'applique le triangle arithmétique.

Il arrive inversement que l'ordre logique strict soit bouleversé pour produire un effet de surprise ou de choc. Cest le cas de l'argument du pari. Si rigoureux qu'en soit le développement, ce n'est qu'à la fin que Pascal propose la question qui aurait dû, dans l'ordre, passer la première, savoir: le pari est-il possible? L'incrédule objecte alors: «j'ai les mains liées et la bouche muette, on me force à parier, et je ne suis pas en liberté, on ne me relâche pas et je suis fait d'une telle sorte que je ne puis croire. Que voulez-vous donc que je fasse?» Dont acte: «Il est vrai...», répond l'apologiste: la question se résout tout de suite par la negative, l'incapacité de croire est une réalité effective, qui traduit tout simplement un manque de grâce. En fait, c'est avant de chercher s'il faut parier qu'il aurait fallu se demander si le pari est possible. Mais si Pascal avait suivi cet ordre, il n'aurait pas pu montrer à quel point le pari, s'il était possible, pourrait être avantageux, et par conséquent combien il est urgent et important d'ôter les obstacles et de plier la machine dans le sens de la conversion.

Pascal se sert parfois d'un ordre pour en annoncer ou en préparer un autre, créant des parallélismes entre démonstrations.

Le Traité des trilignes, dont nous avons décrit plus haut le commencement, comporte deux sections: la première est relative aux sommes d'ordonnées par le biais des ductus plani in planum, la seconde est relative aux sommes de sinus. Les ordonnées d'un triligne sont des perpendiculaires à l'axe d'un triligne issues des divisions égales de cet axe, par exemple les DO du triligne BAH ou les EG du triligne BAC; les sinus naissent des divisions égales de la courbe, comme les IL du triligne BAP. Ils servent chacun à traiter des problèmes différents. Les sommes d'ordonnées engendrent des surfaces planes ou des solides, elles sont donc consacrées à la résolution des problèmes relatifs à leurs mesures. Les sommes de sinus engendrent des surfaces courbes, et sont donc consacrées à des problèmes différents. Entre les propositions de ces deux sections, Pascal institue un ordre similaire: la section sur les sinus suit tout simplement la précédente.

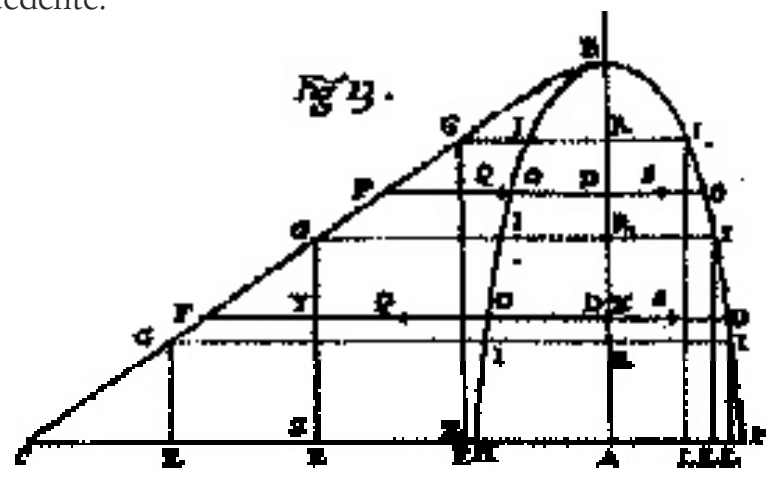


Une Hypothèse préliminaire permet à Pascal de relier l'étude des sinus à celle des ordonnées. Sur le triligne BAP, les sinus (IL.II) sur la base AP naissent des points I des portions II de la courbe, qui sont à la fois égales entre elles et aux portions DD de l'axe BA. On construit la base AC du triligne adjoint BAC égale à la courbe BP supposée rectifiée dans le prolongement de AP. La base AC et la courbe BP sont alors divisées en un même nombre de portions II et EE égales entre elles, et à chaque point E de AC sont élevés les ordonnées EG, chacune égale au sinus IL correspondant (égalité déterminée par la construction des parallèles IRG à PAC). La somme des ordonnées (EG.EE), qui est égale au triligne BAC, est donc l'équivalent de la somme des sinus (IL.II), à ceci près qu'elle est ramenée dans le plan. Pascal est alors en mesure d'appliquer les propriétés démontrées dans la première section pour les sommes d'ordonnées, aux sommes de sinus, dans le même ordre.

La Proposition VI par exemple montre que la somme des arcs BO est égale à la somme des sinus IL. Pascal le prouve en invoquant le double balayage d'une même surface plane, tel qu'il l'a pratiqué dans la Proposition I : en effet, la somme des sinus (IL.II), qui compose une surface courbe, est par construction égale à la somme des ordonnées (EG.EE); celle-ci forme une surface plane, qui est égale à la surface du triligne BAC. Mais par la Proposition I, cette surface BAC est aussi égale à la somme des lignes (DF.DD). Enfin, par construction, cette somme des (DF.DD) est égale à la somme des arcs (BO.DD), qui engendre une surface courbe. Il est donc évident que la somme des sinus IL est égale à celle des arcs BO:

$\sum(\mathrm{DO} . \mathrm{DD})=\sum(\mathrm{DF} . \mathrm{DD})=\sum(\mathrm{EG} . \mathrm{EE})=\sum(\mathrm{IL} . \mathrm{II})$
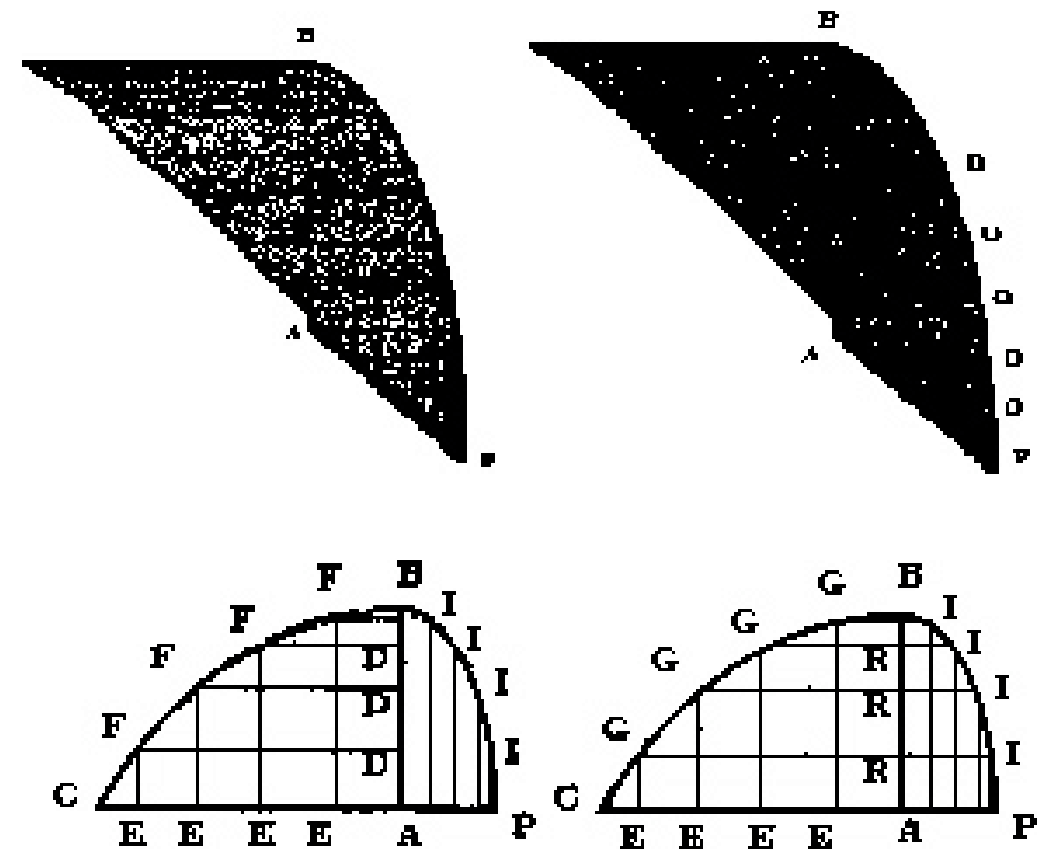
Toutes les propositions suivantes procèderont de même, à ceci près que les puissances des segments iront croissant, des carrés aux cubes et aux carrés-carrés: à chaque fois, l'une des premières propositions, relative aux sommes d'ordonnées, sera invoquée pour établir l'une de celles qui traitent de sinus. Le parallélisme est strict, de sorte que le lecteur, préparé par la première section, peut concentrer son attention sur les particularités des sommes d'arcs et de sinus, qui sont beaucoup plus difficiles à imaginer que les sommes d'ordonnées. Le dédoublement de l'ordre des propositions correspond, d'une certaine manière, à un fractionnement de la difficulté qui facilite l'assimilation des théorèmes.

Les deux premiers chapitres de La pesanteur de la masse de l'air, que nous avons déjà évoqués plus haut, sont à peu près de même farine: Pascal y souligne à plusieurs reprises que la substance et l'ordre en sont directement tirés de L'equilibre des liqueurs, dont il n'est que la transcription analogique, par application des principes qui valent pour les liquides en general au cas particulier de la pression atmosphérique.

De tels parallélismes peuvent donner lieu à de savants entrecroisements. Ainsi dans la version la plus élaborée du Traité de la prédestination, l'exposé de la doctrine de saint Augustin se fait avant celle des Restes des pélagiens, c'est-à-dire des molinistes, puis des calvinistes, il n'y a pas là pourtant une ordonnance simple. En réalité, les trois présentations peuvent être mises en parallèle, thèse par thèse, sous la forme d'un tableau qui évoquerait celle de l'Ecrit à trois colonnes. Pascal veille à ce que le lecteur puisse, pour chaque thèse augustinienne, trouver rapidement la thèse correspondante, mais aussi fort differente, des hérétiques et des pélagiens. Si bien que le lecteur peut lire le traité de deux manières différentes, à son choix: soit à la suite, doctrine par doctrine, soit en confrontant les différents points en controverse, thèse par thèse. Chaque ordre a son sens propre. Exposant à part chacune des doctrines, Pascal en souligne la cohérence interne, surtout dans le cas de l'hérésie calviniste, qui comporte une sorte de logique de l'absurde. D'un autre côté, la mise en parallèle point par point des trois doctrines permet de saisir avec précision les divergences des hérésies par rapport à la vérité catholique.

Il arrive aussi que Pascal ménage des discordances entre l'ordre respectif de traités qui sembleraient à première vue devoir se présenter en parallèle, afin de faire apparaître plus nettement les aspects originaux de chaque théorie. L'ordre du Combinationes par exemple n'est pas calqué sur le Traité du triangle arithmétique, quoique Pascal cherche à en montrer la parenté. L'ordre du Triangle arithmétique est essentiellement fondé sur l'articulation entre égalités et rapports. Le premier mouvement est consacré aux égalités, jusqu'à la Conséquence 11, après quoi Pascal passe aux proportions, est fondé sur la Conséquence 12. Par exemple, la Conséquence seconde, montre que, en tout Triangle arithmétique, chaque cellule est égale à la somme de toutes celles du rang parallèle précédent comprises depuis son rang perpendiculaire jusques au premier inclusivement; elle exprime, sous forme de proposition, la première méthode de génération du triangle arithmétique, et elle sert, par la suite, à établir la correspondance avec les ordres numériques, puisque c'est la règle de formation des nombres figurés. La Conséquence troisième démontre la même chose pour les rangs verticaux. Un peu plus tard, Pascal montre, par la Conséquence quatrième, qu'en tout Triangle arithmétique, chaque cellule diminuée de l'unité est égale à la somme de toutes celles qui sont comprises entre son rang parallèle et son rang perpendiculaire exclusivement. Et ainsi de suite, jusqu'à la Conséquence onzième. La charnière est marquée par un Avertissement qui n'a pas d'équivalent dans la première impression, selon lequel, à «toutes ces conséquences (...) sur le sujet des égalités qui se rencontrent dans le Triangle arithmétique» succèdent «les proportions» entre les nombres qui se trouvent dans les cellules d'une part, et les indices qui figurent sur les côtés du Triangle arithmétique, dont la célèbre Conséquence douzième est le fondement: «En tout Triangle arithmétique, deux cellules contiguës 
étant dans une même base, la supérieure est à l'inférieure comme la multitude des cellules depuis la supérieure jusques au haut de la base à la multitude de celles depuis l'inférieure jusques en bas inclusivement» ${ }^{5}$. Cette proposition commande toutes les suivantes, qui sont de même nature, comme la treizième qui établit que «en tout Triangle arithmétique, deux cellules continues étant dans un même rang perpendiculaire, l'inférieure est à la supérieure comme l'exposant de la base de cette supérieure à l'exposant de son rang parallèle».

On pourrait s'attendre à ce que les traités suivants se conforment à une succession analogue. Ce n'est pas le cas. Il suffit de comparer le diagramme des correspondances avec le traité sur les combinaisons pour voir la différence. D'abord, le Combinationes n'exploite pas toutes les Conséquences du Triangle arithmétique. D'autre part, Pascal estime suffisant de signaler les correspondances entre les deux traités, sans chercher à créer de fausses fenêtres par une mise en parallèle stricte des propositions: dans les Combinaisons, préfère l'ordre qui répond mieux à la logique interne de la matière: la distinction principale s'y fait à partir de la Proposition 7, où il est question, non plus des combinaisons, mais de sommes de combinaisons. La substance des propositions souligne donc les correspondances, mais l'ordre marque l'indépendance des domaines.

Pascal joue parfois avec la règle d'asymétrie, qui veut que, dans un ordre, si un argument $\mathrm{x}$ precède $\mathrm{y}, \mathrm{y}$ ne peut pas précéder $\mathrm{x}$.

Il arrive en effet souvent qu'un ordre soit réversible.

Celui des traités sur la roulette réunis sous le titre de Lettre à Carcavy est de nature analytique: on y montre d'abord qu'en général, la connaissance des solides de rotation demandés par l'auteur du concours dépend de celle des onglets; puis que, si l'on connaît ces onglets dans le cas du cercle générateur, on peut arriver à trouver les mesures cherchées sur la roulette. Ainsi, par transitions successives, l'analyse conduit à des mesures réellement connues, qui donnent le requis. Mais un retournement se produit avec le Traité général de la roulette, qui renoue avec la perspective beuristique polarisée par la résolution des problèmes: Pascal l'a conçu comme un catalogue de références, un connecteur entre les précédents Traités et un guide: il permet, pour obtenir les mesures demandées pour la résolution d'un problème particulier, de retrouver les sommes nécessaires. Par exemple, pour évaluer l'aire du triligne cycloïdal CAF, on sait qu'il faut trouver la somme des ordonnées (YZ.ZZ) de la roulette, qui est égale à celle des lignes mixtes ZMC prise sur le demi-cercle CMF. Or

$\sum[(\mathrm{ZM}+\mathrm{MC}) \mathrm{ZZ}]=\sum(\mathrm{ZM} . \mathrm{ZZ})+\sum(\mathrm{MC} . \mathrm{ZZ})$

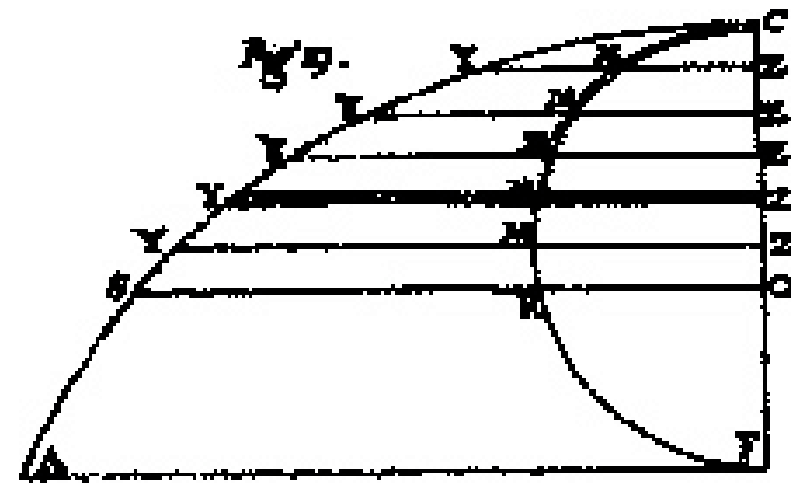

(5) Le texte latin met mieux en relief le type de rapport que Pascal démontre: «Duarum quarumlibet cellularum contiguarum ejusdem basis inferior est ad superiorem ut radix inferioris ad exponentem seriei superioris». 
La valeur de ces sommes est donnée, la première, $\sum$ (ZM.ZZ), dans la Proposition I du Traité des Trilignes, qui montre qu'elle est égale à la portion ORC, et la seconde, la somme d'arcs de cercle $\sum$ (CM.ZZ), par le Traité des arcs de cercle, Propositions 1 et $I I$. Pour les autres problèmes, l'entreprise est laborieuse ${ }^{6}$. De ce fait, à côté de l'itinéraire analytique de la recherche suivi jusque-là par le lecteur, s'en dessine un autre, dans lequel il est amené à calculer par lui-même des résultats précis. Les traités de Dettonville se présentent ainsi comme un ouvrage susceptible d'une double lecture correspondant a un double usage.

De cotte lecture réversible, la Conclusion des Pensées donne un autre exemple, au moment où Pascal avoue «qu'il y a loin de la connaissance de Dieu à l'aimer.» A ce point, il semble que son livre soit devenu inutile, qu'il ait même prouvé sa propre insuffisance. Mais il n'en est rien, car les derniers fragments en dessinent un autre usage. Si la véritable foi peut se passer du raisonnement, lorsque Dieu incline le cœur, il n'est pas pour autant inutile de connaître les raisons de croire, car elles peuvent servir dans la controverse: «J'avoue bien qu'un de ces chrétiens qui croient sans preuves n'aura peut-être pas de quoi convaincre un infidèle, qui en dira autant de soi, mais ceux qui savent les preuves de la religion prouveront sans difficulté que ce fidèle est véritablement inspiré de Dieu, quoiqu'il ne peut le prouver lui-même.» Or quelles seront les preuves qu'un chrétien instruit pourra invoquer pour défendre la foi des ignorants? Ce sont celles mêmes dont l'apologiste a usé: «Dieu ayant dit dans ses prophètes, (qui sont indubitablement prophètes) que dans le règne de J.-C. il répandrait son esprit sur les nations et que les fils, les filles et les enfants de l'Église prophétiseraient il est sans doute que l'esprit de Dieu est sur ceux-là et qu'il n'est point sur les autres.» C'est donc une nouvelle manière d'envisager le problème, un nouveau cadre démonstratif, et un nouvel ordre qui apparaît: il ne s'agit plus du problème zététique de chercher quelle est la vraie religion; il s'agit de justifier la foi d'un autre, ce qui est un problème de confirmation, autrement dit poristique. Les pièces anciennes servent encore, mais avec une autre répartition et une autre signification On peut la résumer en quelques propositions:

1. Les infidèles n'ont aucune preuve de ce qu'ils disent, ce qui renvoie à la liasse Fausseté des autres religions, qui s'oppose à l'argument de Mahomet ««qu'il faut le croire», ce qui ne lui donne aucune autorité, et aussi à Fondements qui montre que «la religion païenne est sans fondement», particulièrement l'Alcoran, qui n'a nulle marque de vérité. A contrario, la religion juive a un fondement admirable: «C'est le plus ancien livre du monde et le plus authentique et au lieu que Mahomet pour faire subsister le sien a défendu de le lire, Moïse pour faire subsister le sien a ordonné à tout le monde de le lire. Et toute religion est de même.»

2. Dieu parle par ses prophètes, qui sont indubitablement prophètes : on reconnaît ici la démonstration de la vérité des prophéties, de l'habileté de Moïse, et les «Preuves des deux testaments à la fois».

3. Dans le règne de J.-C. Dieu répandra son esprit sur les nations et (...) les fils, les filles et les enfants de l'Église prophétiseront, qui renvoie à l'idée que « prophétiser c'est parler de Dieu, non par preuves de dehors, mais par sentiment intérieur et immédiat», et à ce développement connu du lecteur des Pensées: «La plus grande des preuves de J.-C. sont les prophéties. C'est à quoi Dieu a le plus pourvu, car l'événement qui les a remplies est un miracle subsistant depuis la naissance de l'Église jusques à la fin. Aussi Dieu a suscité des prophètes durant 1600 ans et pendant 400 ans après il a dispersé toutes ces prophéties avec tous les juifs qui les portaient dans

(6) Pour en juger, voir l'ouvrage de C. Merker, Le chant du cygne des indivisibles, Presses Universi- 
tous les lieux du monde. Voilà quelle a été la préparation à la naissance de J.-C. dont 1'Évangile devant être cru de tout le monde, il a fallu non seulement qu'il y ait eu des prophéties pour le faire croire mais que ces prophéties fussent par tout le monde pour le faire embrasser par tout le monde.»

4. // est sans doute que l'esprit de Dieu est sur ceux-là. On reconnaît la Conclusion: «Ne vous étonnez pas de voir des personnes simples croire sans raisonnement. Dieu leur donne l'amour de soi et la haine d'eux-mêmes. Il incline leur cœur à croire. On ne croira jamais, d'une créance utile et de foi si Dieu n'incline le cœur et on croira dès qu'il l'inclinera.»

Cet ordre poristique est une invitation à approfondir les points qui peuvent être mis en contestation, et à rechercher de nouvelles raisons de croire.

Le jeu avec la règle d'asymétrie va beaucoup plus loin, lorsqu'il arrive que, de deux arguments $\mathrm{x}$ et $\mathrm{y}$, $\mathrm{x}$ précède $\mathrm{y}$ tout aussi bien que $\mathrm{y}$ précède $\mathrm{x}$, sans qu'il soit possible de choisir par le seul examen de ces termes. Les liasses des Pensées peuvent souvent être associées par couples antithétiques: Vanité et Misère par exemple s'opposent à Grandeur, Divertissement à Philosophes, ou encore Soumission à Excellence ou Fausseté des autres religions à Religion aimable. Mais leur liaison n'est pas toujours de même nature: souvent de conséquence nécessaire, elle est parfois jusqu'à un certain point indifférente. L'ordre qui lie Soumission et Excellence par exemple est strict, de telle manière que l'un des termes précède nécessairement l'autre: Soumission pose les conditions que doit remplir une preuve satisfaisante de la vraie religion, Excellence montre que le christianisme les satisfait. Il n'est pas possible d'inverser la succession. En revanche, dans certains couples, l'ordre n'est pas déterminé par la logique de la démonstration. Cest le cas par exemple des liasses Philosophes et Divertissement. Pascal leur avait assigné une place relative:

«Une lettre de la folie de la science humaine et de la philosophie. Cette lettre avant le divertissement». Mais dans le classement, Philosophes vient après Divertissement. Les deux liasses sont incontestablement corrélatives, dans la mesure où elles montrent, l'une qu'on ne trouve pas le bonheur hors de soi, l'autre qu'on ne le trouve pas non plus en rentrant en soi. Comme ces deux termes symétriques de l'alternative sont également voués à être récusés, peu importe que l'un soit envisagé avant l'autre. Un exemple plus frappant est eolui des deux ensembles consacrés à la grandeur et à la misère de l'homme, dont Pascal a résumé l'ordre en une comptine:

«S'il se vante je l'abaisse.

S'il s'abaisse je le vante.

Et le contredis toujours.

Jusqu'à ce qu'il comprenne

Qu'il est un monstre incompréhensible».

En fait, la succession de la grandeur et de la misère est indifférente, puisqu'on passe toujours naturellement d'une thèse à l'autre par renversement du pour au contre. La seule raison que Pascal a pour commencer par la preuve de la misère de l'homme, c'est qu'il ne serait pas vraiment adroit, pour un auteur qui prétend combattre l'amour-propre, de commencer par persuader le lecteur de sa grandeur, chose dont il n'est en général que trop convaincu. C'est donc la tactique de la persuasion, non la logique, qui a décidé.

Le jeu est plus complexe lorsque l'ordre comporte une dualité latente dont les fils se nouent. Dans l'esprit de Pascal, l'apologie pouvait être présentée sous deux aspects différents. 
1. Partie. Misère de

l'homme sans Dieu.

2. Partie. félicité de

l'homme avec Dieu.

autrement

1. Part. Que la nature

est corrompue, par la Nature

même.

2. Partie. qu'il y a un

Réparateur par l'Écriture.

Ces deux plans ne sont pas exclusifs l'un de l'autre: ce sont deux manières de présenter une même succession de preuves. Le premier met l'accent sur deux contrastes, misère de l'bomme sans Dieu et felicité avec Dieu d'une part, et corruption et réparation d'autre part. Le deuxième ajoute à la considération des points à démontrer, que la nature est corrompue et qu'il y a un réparateur, la considération de la manière de le prouver, par la Nature, et par l'Ecriture. Sous le parallélisme apparent, la différence de niveau est évidente entre les deux ordres: le premier répond à ce que, dans L'esprit géométrique, Pascal appelle l'analyse, appliquée à la nature morale et psychologique de l'homme; le second répond au point de vue de la critique, et de la vérification de la conformité des preuves aux règles de la démonstration. Cette double perspective est présente dans les textes les plus élaborés des Pensées. En effet, dans la prosopopée de la Sagesse de Dieu par exemple, Pascal ne se contente pas seulement d'expliquer comment la Révélation rend compte de l'état actuel de la condition humaine; il montre aussi que la transition d'une vérité philosophique à une vérité révélée ne contrevient pas aux exigences légitimes de la pensée humaine, autrement dit que la Sagesse de Dieu ne s'impose pas de manière tyrannique. Preuve et réflexion sur la légitimité de la preuve, démonstration et critique de la démonstration sont menées de pair, et placées pour ainsi dire en même ordonnance.

De tels parallélismes engendrent l'apparition de nexus rationum ${ }^{7}$, c'est-à-dire d'entrelacements de raisons : celles-ci ne sont pas simplement enfilées bout à bout en série : chaque raison intervient dans plusieurs argumentations à la fois, de sorte que celles-ci retentissent les unes sur les autres, de sorte que chaque démonstration a des effets dans plusieurs domaines.

Ces nœuds dans l'ordre sont fréquents dans l'oeuvre mathématique. Les Lettres de A. Dettonville en donnent un autre exemple remarquable, lorsque Pascal cherche la mesure du bras d'un triligne sur sa base, c'est-à-dire de la distance qui sépare sa base AC de son centre de gravité: cette mesure s'obtient par la somme triangulaire des ordonnées (DF.DD) à l'axe $\mathrm{AB}, \mathrm{T}(\mathrm{DF} . \mathrm{DD})$. Le raisonnement s'énonce donc comme suit:

$$
\begin{aligned}
& \mathrm{T}_{\mathrm{A}}(\mathrm{DF} \cdot \mathrm{DD})=\mathrm{ABC} \cdot \mathrm{YE} \\
& \mathrm{YE}=\frac{\mathrm{T}_{\mathrm{A}}(\mathrm{DF} \cdot \mathrm{DD})}{\mathrm{ABC}}
\end{aligned}
$$



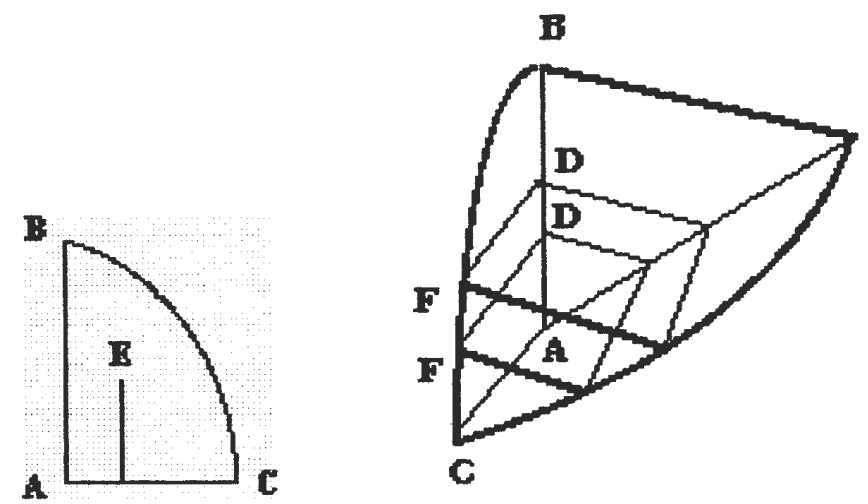

Du même coup, Pascal résout un autre problème, celui de la cubature de l'onglet sur la base AC du triligne BAC, ou de son double onglet. La somme des (DF.DA.DD), c'est-à-dire, puisque chaque DA est égal au segment FM ou FN correspondant, des rectangles (DF.FM.DD) ou (DF.FN.DD) est égale à la somme triangulaire des ordonnées (DF.DD).

Doubl.onglet ${ }_{\mathrm{AC}} \mathrm{ABC}=\sum\left(\mathrm{EG}{ }^{2} \mathrm{EE}\right)=2 \sum$ (DF.DA.DD $)=2 \mathrm{~T}_{\mathrm{AC}}(\mathrm{DF} \cdot \mathrm{DD})$
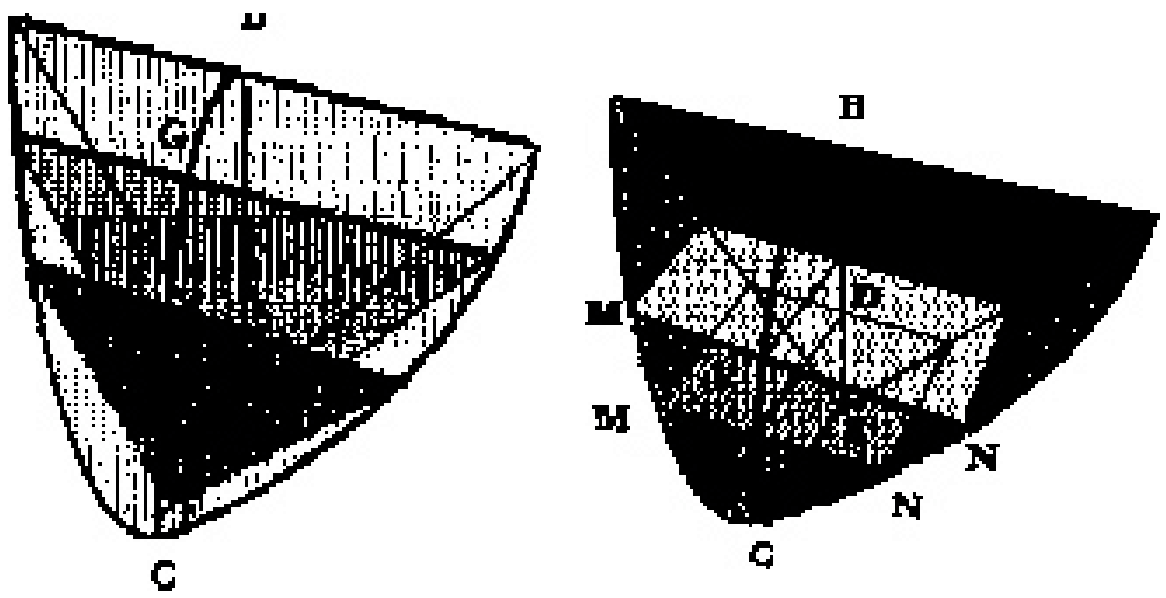

Ce sont donc deux problèmes de genre très différent, l'un sur la mesure de la dimension d'un solide, l'autre sur un centre de gravité, qui sont résolus en même temps.

(7) Voir là-dessus M.Gueroult, Descartes selon l'ordre des raisons, Paris, Aubier, 1968, I, p. 237. Dans les Méditations, M. Gueroult distingue plusieurs nœuds, de plus en plus complexes : d'abord dans le Cogito, puis dans la question de la valeur objective des idées (Méditation III), enfin dans la VIe Méditation, qui est un nexus de nexus. 
Pascal effectue une semblable réduction dans les Pensées, lorsqu'il se propose de fournir les «Preuves des deux testaments à la fois». Le raisonnement est le suivant: «Pour prouver d'un coup tous les deux il ne faut que voir si les prophéties de l'un sont accomplies en l'autre. Pour examiner les prophéties il faut les entendre. Car si on croit qu'elles n'ont qu'un sens il est sûr que le Messie ne sera point venu, mais si elles ont deux sens il est sûr qu'il sera venu en J.-C. Toute la question est donc de savoir si elles ont deux sens.» Au départ, il s'agit donc de résoudre un problème de vérité, savoir si les prophéties de l'Ancien Testament sont accomplies dans le Nouveau. Mais cette question présuppose la résolution d'une autre, car «pour examiner les prophéties il faut les entendre». Celle-ci en enferme à son tour deux: l'une, de fait, de savoir «si elles ont deux sens»; la seconde, d'interprétation, de savoir quel est le sens spirituel qui se cache derrière le sens charnel. Cette dernière question a une portée pour la vérification des prophéties, puisque c'est seulement si les prophéties «ont deux sens il est sûr qu'il sera venu en J.-C.»; mais elle ouvre aussi un nouveau champ d'argumentation, relatif au caractère spirituel de la religion chrétienne, par opposition à la religion juive, ou à toute autre religion. Ainsi, la preuve des deux testaments à la fois enferme-t-elle un nœud où les propositions se suivent avec rigueur, mais où chacune a des retentissements divers dans l'Apologie.

Le concept de tyrannie engendre un nexus rationum encore plus complexe. Il se degage progressivement des rédactions successives du fragment Laf. 58, Sel. 9192. Sa signification est double. En premier lieu, il est directement lié à la substance de l'argumentation morale, dans la mesure où il répond au concept de misère, que Jean Mesnard définit comme l'état la volonté privée du pouvoir ${ }^{8}$. La tyrannie consiste à «vouloir avoir par une voie ce qu'on ne peut avoir que par une autre», c'està-dire à employer des moyens qui appartiennent à un ordre pour obtenir ce qui relève d'un autre. C'est une manière abusive de chercher remède à la misère. Dans ce contexte, les notions connexes sont celles d'usurpation et de violence. Le concept de tyrannie induit des argumentations sur la faiblesse de l'homme, dans la mesure où il entre dans la nature de moyens inadaptés de manquer leur but: la tyrannie est toujours vouée à l'échec et au ridicule. Mais ce concept a aussi sa place dans le fil critique de l'apologie: il permet de définir ce qui est insupportable à l'homme, soit du point de vue de l'esprit, soit du point de vue du cœur, autrement dit il doit aussi servir de critère d'admission ou de rejet des doctrines qui prétendent enfermer la vérité de la condition humaine. Ce que l'homme ne peut admettre, du point de vue de l'esprit, c'est qu'on lui impose une vérité par la force, sans laisser place à l'exercice de la raison, autrement dit qu'on cherche à imposer une «superstition». Et du point de vue du bonheur, il ne peut supporter qu'on lui impose un bien qui ne soit pas le souverain bien, ou encore pire, une doctrine qui le réduise au désespoir.

Après Misère, ce concept fera plusieurs apparitions dans l'une ou l'autre fonction, mais toujours de manière decisive. Il réapparaît dans le discours de la Sagesse de Dieu, lorsqu'elle déclare que, pour ne pas s'imposer de manière tyrannique, elle prétend rendre compte à l'homme de son état par la doctrine de la corruption. Il est sous-jacent à Soumission et usage de la raison, qui accorde à la pensée humaine son exercice libre à partir des principes nouveaux de la Révélation. Il apparaît dans Fausseté, dans la manière dont Pascal montre que Mahomet au contraire impose sa religion par violence. Enfin, il est dans Morale chrétienne, avec l'idée de membre du corps, qui exclut de la morale chrétienne la tyrannie totalitaire. Dans chacune de 
ces interventions, il sert de critère de filtrage, ou de discernement de la vérité et de l'erreur.

Le nœud des raisons est particulièrement visible à la charnière de l'Apologie, qui repose sur une sorte de preuve apagogique. Le nerf de la démonstration est d'ordre critique, dans la mesure où il tire la conséquence du cercle vicieux où conduisent les philosophies humaines: les premiers chapitres ont montré que les contrariétés de la nature sont telles que de la grandeur on conclut la misère et inversement, sans fin. Cette impasse oblige à remettre en cause les fondements du problème: il faut récuser les principes fondés sur la raison naturelle des philosophes, puisque ce sont eux qui engendrent les apories auxquelles on s'est heurté, et poser les bases d'une problématique renouvelée, en acceptant des principes qui viennent d'une autre instance, savoir d'une révélation de nature religieuse. $A P R$ en formule donc les termes, en posant les conditions auxquelles toute religion, pour pouvoir sérieusement prétendre exprimer la vérité, doit satisfaire. La première condition est d'ordre critique: cette vérité ne doit pas s'imposer de manière tyrannique, il faut qu'elle réponde à une exigence de la raison elle-même. Philosophes et Divertissement montrent qu'on ne doit pas demander à l'intérieur, comme le prétendent les Stoïciens, ce qu'on ne trouve pas à l'extérieur, comme le montre l'échec des conduites de diversion. APR et Souverain Bien présentent les critères auxquels doit répondre la solution cherchée, Excellence confirme que la voie proposée par la religion chrétienne est conforme à ces exigences. Mais d'autres conditions ont été fournies par l'analyse morale, qui demeure valable de ce point de vue : la vraie religion doit déclarer le double aspect de misère et de grandeur de l'homme, et le besoin de vrai et de bien qui l'habite, et ensuite en expliquer la raison. «Après avoir entendu toute la nature de l'homme il faut pour faire qu'une religion soit vraie qu'elle ait connu notre nature. Elle doit avoir connu la grandeur et la petitesse et la raison de l'une et de l'autre»; «la vraie religion doit avoir pour marque d'obliger à aimer son Dieu. Cela est bien juste et cependant aucune ne l'a ordonné, la nôtre l'a fait. Elle doit encore avoir connu la concupiscence et l'impuissance, la nôtre l'a fait. Elle doit y avoir apporté des remèdes, l'un est la prière. Nulle religion n'a demandé à Dieu de l'aimer et de le suivre.» Enfin Fausseté envisage les mauvaises réponses qu'on peut donner au problème. On constate donc que, dans la question, les deux fils argumentatifs, moral et critique, forment un nœud si serré qu'on ne peut le défaire sans défaire toute le démonstration.

Mais il ne faut pas croire que la différence de niveau entre l'argumentation morale et l'argumentation critique crée une hétérogénéité dans l'ordre. Le nexus rationum assure l'unité de l'ensemble. En effet, l'argumentation critique a sa place dans l'ordre moral: céder à la tyrannie de la superstition, par exemple croire un Mahomet parce qu'il tue, est une preuve supplémentaire de la misère dont l'homme est capable; inversement, refuser de soumettre la raison là où elle devrait le faire, comme le font certains libertins, c'est aussi une preuve de la présomption de l'homme, c'està-dire de sa misère. Mais il faut aussi considérer que l'argumentation critique, qui vise à établir les conditions auxquelles une doctrine philosophique ou religieuse peut prétendre représenter la vérité n'a de sens que dans la mesure où elle prolonge l'argumentation morale: c'est parce que l'homme constate sa misère, c'est-à-dire le caractère inadéquat à sa nature des morales qu'on lui propose qu'il est conduit à imposer à toute doctrine des conditions srictes pour être acceptable. Les deux ordres de raisons sont toujours distincts, mais ils convergent au bout du compte comme les parallèles arguésiennes.

Le jeu sur l'ordre ou avec l'ordre engendre la polyvalence des démonstrations sans en compromettre l'unité. Les précédentes remarques ne sauraient prétendre épuiser la question de l'ordre chez Pascal, dont on saisit à présent la complexité. Ce serait une erreur de réduire la pratique pascalienne aux règles de l'esprit géométrique, 
règles fondamentales et nécessaires, mais non suffisantes. La clé de sol n'est pas la partition entière. Les principes élémentaires posés, il reste à en faire bon usage, selon les exigences subtiles de l'art de persuader. Dans les œuvres scientifiques comme dans les Pensées, l'ordre est complexe, muable, multiple et subtil. Quoiqu'en dise Claudel, ce n'est pas le désordre seul qui fait les délices de l'imagination: l'ordre bien mené en fait tout autant ?

DOMINIQUE DESCOTES

(9) Il faudrait encore, pour être complet, montrer en quoi l'ordre est aussi, chez Pascal, le reflet de l'invention. Il n'est pas question de l'entreprendre dans le cadre restreint de la présente étude. On se contentera de renvoyer, sans insister, à l'étude du manuscrit du fragment Infini rien par G. Brunet et H. Gouhier, aux travaux de Y. Maeda, de P. Ernst et de P. Sellier sur les Pensées, ou à l'étude des traités sur la roulette par P. Costabel, K. Hara, J. Mesnard et moi-même, pour apercevoir les amples perspectives qui s'ouvrent à la recherche de ce côté-là. 